\title{
Higher Magnification Microsurgical Repair of Donor Artery Dissection in Superficial Temporal Artery to Middle Cerebral Artery Anastomosis
}

\author{
-Technical Note-
}

\author{
Nobuhisa MAtSumura, ${ }^{1}$ Takashi SHIBATA, ${ }^{1}$ Hironaga KAMIYAMA, ${ }^{1}$ \\ Takahiro TOMITA, ${ }^{1}$ Soushi OKAMOTO, ${ }^{1}$ Michiya KUBO, ${ }^{1}$ and Yukio HORIE ${ }^{1}$ \\ ${ }^{1}$ Department of Neurosurgery, Stroke Center, Saiseikai Toyama Hospital, Toyama, Toyama
}

\begin{abstract}
Donor artery dissection is a known cause of technical failure in microvascular anastomosis. A method for detection and direct repair of donor artery dissection before superficial temporal artery (STA) to middle cerebral artery (MCA) anastomosis is described using a high magnification operating microscope (maximum 50.4 $\times$ magnification). Before STA-MCA anastomosis, the stump of the STA is stained using methylrosaniline chloride (pyoctaninum blue) and is observed under higher magnifications. Microsurgical suturing of the arterial dissection is performed before the anastomosis procedure under the high magnification microscope. This method was used in two patients with symptomatic hemodynamic cerebrovascular occlusive disease. Postoperative angiography revealed good patency and no complications occurred. This method may be useful for detection and direct repair of arterial dissection in small vessel walls before STA-MCA anastomosis.
\end{abstract}

Key words: bypass, arterial dissection, superficial temporal artery, superficial temporal artery to middle cerebral artery anastomosis, microvascular anastomosis

\section{Introduction}

Superficial temporal artery (STA) to middle cerebral artery (MCA) anastomosis is usually performed between scalp and cortical arteries of $0.5-2.0 \mathrm{~mm}$ in diameter at $10-25 \times$ magnification. ${ }^{6,8}$ Microvascular anastomosis requires great care in performing atraumatic manipulations of small vessels and correct suturing of arterial walls for good technical results. Arterial dissection or other pathological changes in a small arterial wall are known causes of technical failure in microvascular anastomosis. ${ }^{1,3)}$

Here, we describe a method for higher magnification microsurgical repair of the donor artery dissection before the anastomosis procedure for safer and more accurate STA-MCA anastomosis.

\section{Methods}

A high magnification operating microscope (MM50/ YOH; Mitaka Khoki Co., Ltd., Mitaka, Tokyo) is used with the combination of a $10 \times$ eyepiece and 200 ,

Received May 12, 2011; Accepted August 17, 2011
250 , and $300-\mathrm{mm}$ objective lenses to provide a range of final magnification from 2.9 to $50.4 \times$. This new lens design enables very high resolution (resolution of 7-8 $\mathrm{mm}$ at $50.4 \times)$ and good depth of focus (1.0 $\mathrm{mm}$ at $50.4 \times$ ), producing a very clear image of the object for practical use in operative procedures.

The STA is exposed by atraumatic manipulation from the scalp skin and is cut off at the distal portion. The stump of the STA is stained using $0.5 \%$ pyoctaninum blue solution (methylrosaniline chloride). The stained wall of the donor artery is observed at higher magnifications using this operating microscope. If any clear donor artery dissection is detected, microsurgical repair of the dissection is performed by suturing using 11-0 nylon sutures with an $80-\mathrm{mm}$ diameter needle at higher magnifications before the anastomosis procedure. Microvascular anastomosis is then performed using 10-0 nylon sutures with a $120-\mathrm{mm}$ diameter needle at higher magnifications.

We have used this method for two patients with symptomatic hemodynamic cerebrovascular occlusive diseases. No patient experienced postoperative neurological deficits and postoperative magnetic 


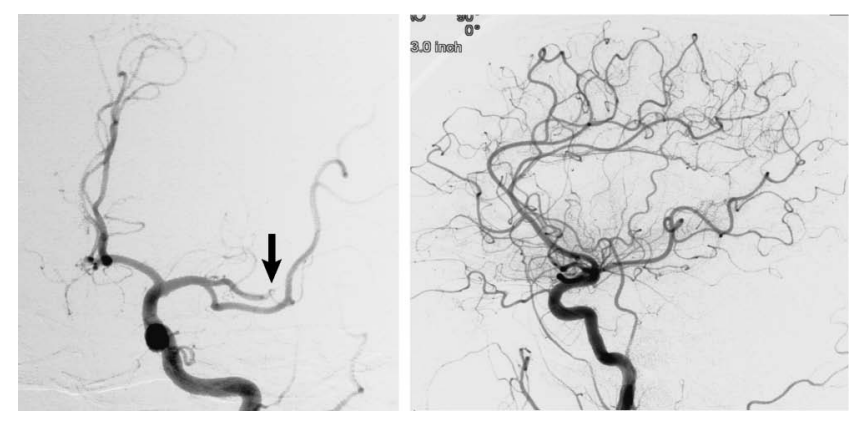

Fig. 1 Preoperative anteroposterior (left) and lateral (right) left common carotid angiograms demonstrating the occluded middle cerebral artery (arrow).
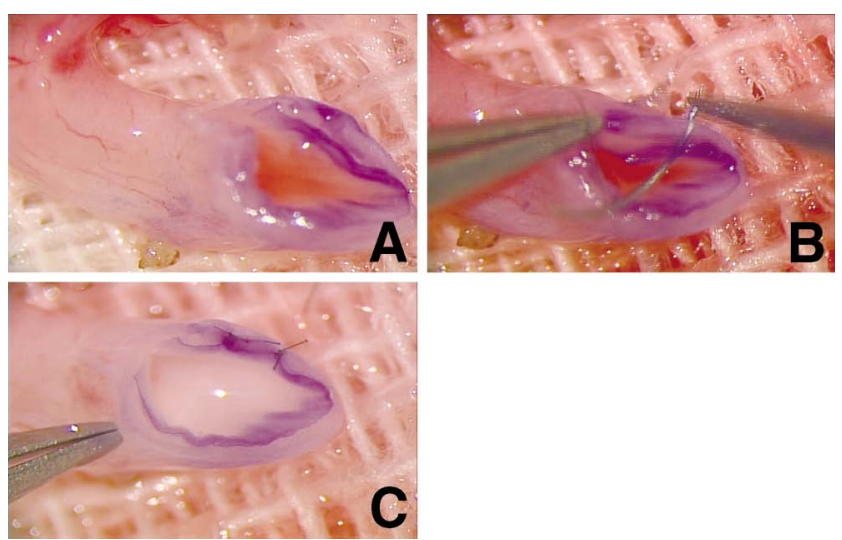

Fig. 2 Intraoperative photographs at $50.4 \times$ magnification obtained with the high magnification operating microscope showing arterial dissection in the donor arterial wall. A: Stump of the donor artery was stained using $\mathbf{0 . 5 \%}$ pyoctaninum blue solution. B: Microsurgical suturing was performed using 11-0 sutures. C: Arterial dissection was repaired with two stitches.

resonance imaging showed no new ischemic lesions. Postoperative angiography revealed good patency.

Representative case: A 61-year-old man with a history of untreated hypertension and diabetes mellitus was referred to our hospital with mild right hemiparesis. Radiological examinations showed small left frontal subcortical infarctions and hemodynamic cerebrovascular ischemia in the left frontoparietal lobe. Cerebral angiography revealed occlusion of the left MCA (Fig. 1). The indications for STA-MCA anastomosis were determined according to the inclusion criteria of the Japanese Extracranial-Intracranial Bypass Trial (JET Study). The patient underwent left STA-MCA anastomosis. The parietal branch of the left STA was exposed by

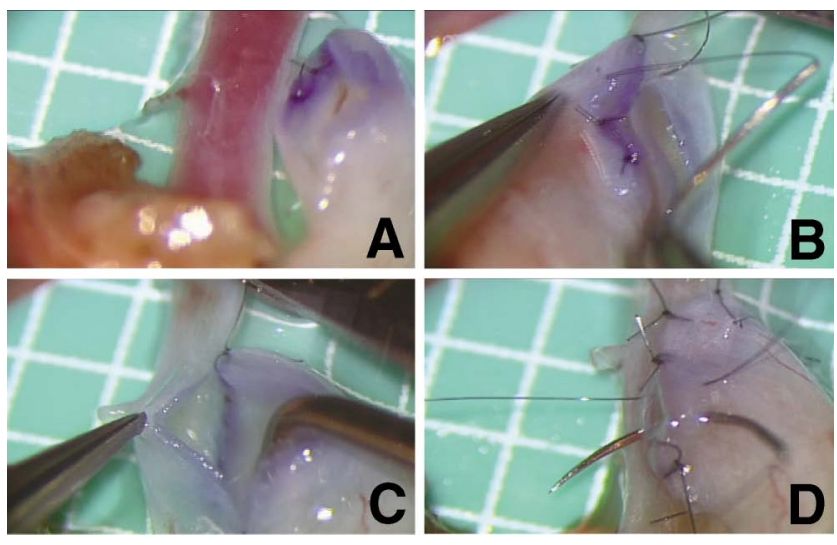

Fig. 3 Intraoperative photographs obtained with the high magnification operating microscope showing an anastomosis on a silicone rubber sheet. One scale unit on the sheet is $1.0 \mathrm{~mm}$. A: Microsurgical view of the repaired donor artery and the recipient cortical artery. B: Microvascular suturing at $50.4 \times$ magnification was performed using 10-0 nylon suture for the anastomosis. C: Microsurgical suturing was correct through the contralateral inner lumen. The 11-0 sutures were tied outside the intimal lumen. D: End-to-side anastomosis was sutured completely.

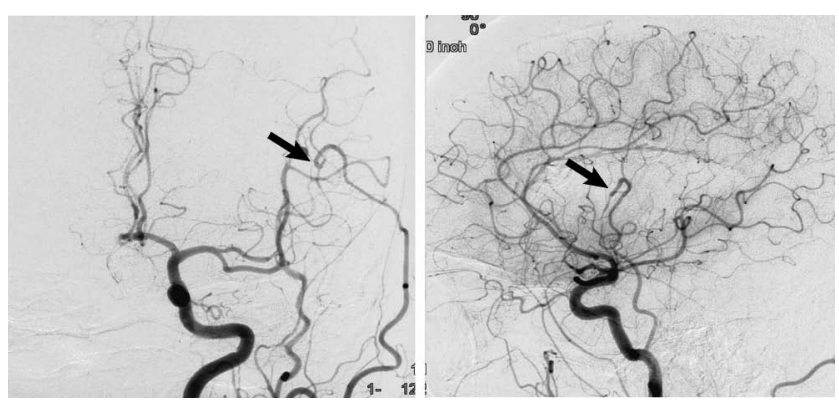

Fig. 4 Postoperative anteroposterior (left) and lateral (right) left common carotid angiograms showing good patency of the left superficial temporal artery to middle cerebral artery anastomosis (arrow).

atraumatic manipulation from the scalp skin and was cut off at the distal portion from the skin incision. This donor artery was $2.0 \mathrm{~mm}$ in external diameter. The stump of the donor artery was trimmed and stained clearly. Higher magnification operative views enabled observation of the three layers of the stained stump of the donor arterial wall clearly. Intraoperative findings showed a donor artery dissection at $50.4 \times$ magnification (Fig. $2 \mathrm{~A}$ ). We tried to recut the stump, but the arterial dissection was still observed. Microsurgical direct repair was performed using two stitches with 11-0 nylon suture at $50.4 \times$ magnification (Fig. 2B, C). This recipient cortical ar- 
tery was $1.0 \mathrm{~mm}$ in external diameter. End-to-side anastomosis was performed using 12 stitches with 10-0 nylon suture inside the tied 11-0 sutures, and appropriate suturing of the STA and MCA endothelial surfaces was confirmed at higher magnifications (Fig. 3). Postoperative magnetic resonance imaging revealed no additional findings. Postoperative angiography revealed good patency (Fig. 4). No postoperative events occurred and the patient was discharged without neurological deficits.

\section{Discussion}

Staining using pyoctaninum blue solution has been reported as a technique for visualizing the ostium of an arteriotomy for the treatment of moyamoya diseases. ${ }^{2}$ This method is effective to detect a cut line in a very small recipient artery in microvascular anastomosis. Selection of a standard operating microscope could also allow detection and treatment of the donor artery dissection. However, careful observation and manipulation of very small arteries or arterial walls is still necessary using standard operating microscopes.

The standard operating microscope now utilized in neurosurgery has a range of practical final magnification from $1.5 \times$ to about $25 \times .^{7,8)}$ Appropriate observation and manipulation at higher magnifications are very important, and optimal organization of the operative field is required. Higher magnification microsurgery is technically more useful for the finest anastomoses of the brain,4) but may also require special technical training and experience.5) However, the practical final magnifications of 30-50 $\times$ provide super-magnified operative views in neurosurgery which would facilitate safe treatment to eliminate mismatching of the small arterial wall.

The present method allows safer and more precise microvascular anastomosis techniques. The working space for repairing the donor artery dissection is not limited by other stitches in the anastomosis procedure, and repairing the donor artery dissection is not performed during clamping of the recipient artery. We recommend detection and direct repair of arterial dissection in the small vessel walls before STA-MCA anastomosis.

\section{References}

1) Diaz FG, Chason J, Shrontz C, Ausman JI, Dujovny M: Histological structural abnormalities of superficial temporal arteries used for extracranial-intracranial anastomosis. J Neurosurg 57: 328-333, 1982

2) Kamiyama H, Takahashi A, Houkin K, Mabuchi S, Abe $\mathrm{H}$ : Visualization of the ostium of an arteriotomy in bypass surgery. Neurosurgery 33: 1109-1110, 1993

3) Levinthal R, Gregorius FK: Intimal dissection of the superficial temporal artery. Surg Neurol 9: 268-269, 1978

4) Matsumura N, Hayashi N, Kamiyama H, Kubo M, Shibata T, Okamoto S, Horie Y, Hamada H, Endo S: Microvascular anastomosis at 30-50 $\times$ magnifications (super-microvascular anastomosis) in neurosurgery. Surg Neurol Int 2: 6, 2011

5) Matsumura N, Shibata T, Umemura K, Nagao S, Horie Y: Extracranial-intracranial bypass surgery at high magnification using a new high-resolution operating microscope: technical note. Surg Neurol 72: 690-694, 2009

6) Newell DW, Vilela MD: Superficial temporal artery to middle cerebral artery bypass. Neurosurgery 54: 1441-1448, 2008

7) Sekhar LN, de Oliverira E: Cranial Microsurgery: Approaches and Techniques. New York, Thieme, 1999

8) Yaşargil MG: Microsurgery Applied to Neurosurgery. Stuttgart, Georg Thieme Verlag, 1969

Address reprint requests to: Nobuhisa Matsumura, MD, PhD, Department of Neurosurgery, Saiseikai Toyama Hospital, 33-1 Kusunoki, Toyama 931-8533, Japan. e-mail: sanataka@pk.ctt.ne.jp 